\title{
AUTOMORPHISMS OF OPERATOR ALGEBRAS
}

\author{
BY RICHARD V. KADISON ${ }^{1}$ AND JOHN R. RINGROSE
}

Communicated by P. R. Halmos, June 29, 1966

1. Introduction. We describe certain results concerning the structure of the group $\alpha(\mathfrak{H})$ of automorphisms of a $C^{*}$-algebra $\mathfrak{A}$. They will appear, together with their proofs, in Communications in Mathematical Physics.

All mappings of $\mathfrak{A}$ we consider preserve the ${ }^{*}$ structure. With $\phi$ a faithful representation of $\mathfrak{A}$ on the Hilbert space $\mathfrak{F}$, we say that an automorphism $\beta$ of $\phi(\mathfrak{H})$ is weakly-inner when there is a unitary operator $U$ in the strong-operator closure $\phi(\mathfrak{H})^{-}$of $\phi(\mathfrak{I})$ such that $\beta(A)=U A U^{*}$ for all $A$ in $\phi(\mathfrak{A})$. We denote by $\iota_{\phi}(\mathfrak{H})$ the subgroup of $\alpha(\mathfrak{U})$ consisting of those $\alpha$ for which $\phi \alpha \phi^{-1}$ is weakly-inner, by $\epsilon_{\phi}(\mathfrak{U})$ those $\alpha$ such that $\phi \alpha \phi^{-1}$ extends to an automorphism of $\phi(\mathfrak{H})^{-}$, by $\sigma_{\phi}(\mathfrak{A})$ those $\alpha$ such that there is some unitary operator $U$ on $\mathfrak{H}$ for which $\phi \alpha \phi^{-1}(A)=U A U^{-1}$ when $A$ lies in $\phi(\mathfrak{H})$ and by $\pi(\mathfrak{A})$ the intersection of all $\iota_{\phi}(\mathfrak{U})$. We refer to the elements of $\sigma_{\phi}(\mathfrak{U}), \epsilon_{\phi}(\mathfrak{A})$ and $\pi(\mathfrak{H})$ as the spatial, extendable, permanently weakly (for brevity, $\pi-$ ) inner automorphisms, respectively, (of $\phi(\mathfrak{I})$ in the first two instances and $\mathfrak{A}$ in the last). We denote by $\iota_{0}(\mathfrak{A})$ the group of inner automorphisms of $\mathfrak{A}$.

The group $\alpha(\mathfrak{I})$ consists of operators on the Banach space $\mathfrak{A}$ each of which is isometric so that $\alpha(\mathfrak{U})$ acquires a norm (or metric) topology as a subset of the bounded operators on $\mathfrak{A}$. We consider $\alpha(\mathfrak{H})$ and the various subgroups defined as provided with this topology. We denote by $\gamma(\mathfrak{R})$ the connected component of the identity element $\iota$ of $\alpha(\mathfrak{U})$.

2. The automorphism group. It has been proved recently [3], [4], [5] that each derivation $\delta$ of a $C^{*}$-algebra $\mathfrak{A}$ acting on the Hilbert space $\mathfrak{H C}$ is weakly inner-that is, there is a $B$ in $\mathfrak{A}^{-}$such that $\delta(A)$ $=B A-A B$ for all $A$ in $\mathfrak{A}$. Combining this result with those of Nagumo-Yosida and some series computations, we have:

Lemma 1. Each norm-continuous one-parameter subgroup of $\alpha(\mathfrak{H})$ lies in $\pi(\mathfrak{H})$.

Spectral theory and von Neumann algebra considerations yield:

Lemma 2. If $\alpha$ is an inner automorphism of a von Neumann algebra

${ }^{1}$ Completed with the partial support of NSF and ONR. 
Q such that $\|\alpha-\imath\|<2$; then there is a unitary operator $U$ in $R$ with spectrum $\sigma(U)$ in the half-plane $\left\{z: \operatorname{Re} z \geqq \frac{1}{2}\left(4-\|\alpha-\iota\|^{2}\right)^{1 / 2}\right\}$ such that $\alpha(A)=U A U^{*}$ for all $A$ in $R$.

Geometrically, $\sigma(U)$ is contained in the arc of the unit circle containing 1 with endpoints midway between 1 and the points of the circle at distance $\|\alpha-\imath\|$ from 1 . Employing the automorphism $\beta$ of the factor of type $\mathrm{II}_{1}$ associated with the free group on three generators arising from a cyclic permutation of these generators, we see that the conclusion of the lemma fails in this case $(\|\beta-\imath\|=2)$.

Disjoint representation together with von Neumann algebra methods permit us to prove:

Lemma 3. If $\alpha$ is an automorphism of the $C^{*}$-algebra $\mathfrak{A}$ acting on $\mathfrak{H C}$ such that $\|\alpha-\iota\|<2$, then $\alpha$ extends to an automorphism $\bar{\alpha}$ of $\mathfrak{A}-$ such that $\|\bar{\alpha}-\imath\|=\|\alpha-\imath\|<2$.

Techniques of the theory of operator-valued analytic functions combined with Banach algebra methods (much like those of [2], or directly, using [2; Corollary 3]) give:

Lemma 4. If $\mathfrak{A}$ is a $C^{*}$-algebra and $U$ a unitary operator acting on $\mathfrak{H C}$ such that $\alpha(A)=U A U^{*}$ lies in $\mathfrak{A}$ for all $A$ in $\mathfrak{A}$ and $\operatorname{Re} \lambda>0$ for each $\lambda$ in $\sigma(U)$ then $\alpha$ lies on a norm-continuous one-parameter subgroup of $\alpha(\mathfrak{H})$ and is $\pi$-inner.

The outer automorphism of the $\mathrm{II}_{1}$ factor associated with the free group on two generators and the unitary operator (multiplied by $(-1)^{1 / 2}$ ) arising from interchanging those generators illustrate the fact that the conclusion of the above lemma need not hold if the hypothesis is weakened to allow $\sigma(U)$ to lie in the closed right halfplane. Note that Lemmas 2 and 4 allow us to conclude that Lemma 2 holds with "inner" deleted and $R$ a $C^{*}$-algebra (replacing the second occurrence of $R$ by $R^{-}$).

The preceding results and some representation theory for $C^{*}$ algebras allow us to conclude:

Theorem 5. If $\alpha$ is an automorphism of a $C^{*}$-algebra $\mathfrak{A}$ and $\|\alpha-\imath\|$ $<2$ then $\alpha$ lies on a norm-continuous one-parameter subgroup of $\alpha(\mathfrak{R})$. Such subgroups generate $\gamma(\mathfrak{H})$ (as a group); and $\gamma(\mathfrak{H})$ is an open subgroup of $\alpha(\mathfrak{I})$, each element of $\gamma(\mathfrak{I})$ being $\pi$-inner. Since

$$
\gamma(\mathfrak{I}) \subseteq \pi(\mathfrak{I}) \subseteq \iota_{\phi}(\mathfrak{I}) \subseteq \sigma_{\phi}(\mathfrak{I}) \subseteq \epsilon_{\phi}(\mathfrak{I}) \subseteq \alpha(\mathfrak{I}),
$$

all these subgroups are open as well as closed in $\alpha(\mathfrak{H})$. Moreover, $\gamma(\mathfrak{H})$, $\pi(\mathfrak{H})$ and $\iota_{0}(\mathfrak{H})$ are normal subgroups of $\alpha(\mathfrak{H})$; while $\iota_{\phi}(\mathfrak{H}), \sigma_{\phi}(\mathfrak{I})$ and 
$\boldsymbol{\epsilon}_{\phi}(\mathfrak{U})$ need not be. In addition $\iota_{\phi}(\mathfrak{U})$ is a normal subgroup of $\epsilon_{\phi}(\mathfrak{H})$, though $\sigma_{\phi}(\mathfrak{U})$ need not be.

As an immediate consequence, we have:

COROLLARY 6. Each (norm) continuous representation of a connected topological group in $\alpha(\mathfrak{H})$ has range consisting of $\pi$-inner automorphisms of $\mathfrak{A}$.

COROLlARY 7. If $\mathfrak{U}$ has a faithful representation $\phi$ as a von Neumann algebra then $\iota_{0}(\mathfrak{H})=\gamma(\mathfrak{U})=\pi(\mathfrak{U})=\iota_{\phi}(\mathfrak{U})$; and each element of $\gamma(\mathfrak{U})$ lies on some norm-continuous one-parameter subgroup of $\alpha(\mathfrak{U})$.

The situation described by the results of this section is sharply in contrast with that which prevails when $\alpha(\mathfrak{A})$ is provided with topologies weaker than its norm topology. In [1] Blattner shows that each locally compact group satisfying a countability axiom has a strongly-continuous unitary representation by operators inducing outer automorphisms of a factor of type $\mathrm{II}_{1}$ (except for the unit element).

3. Examples. The examples which follow illustrate, for specific $C^{*}$-algebras, various automorphism phenomena not described by the results of $\S 2$.

(a) If $\mathfrak{A}$ has a norm-dense subalgebra which is the ascending union of * subalgebras (with the unit of $\mathfrak{U}$ ) $\mathfrak{M T}_{n}$ isomorphic to the algebra of operators on $2^{n}$-dimensional Hilbert space, the automorphism $\alpha$ of $\mathfrak{A}$ which is (successive) transposition about both diagonals of each $\mathfrak{T M}_{n}$ is weakly-inner in some (irreducible) representation of $\mathfrak{A}$ on $\mathfrak{L}_{2}(0,1)$ provided with Lebesgue measure with $[0,1]$ partitioned by dyadic rationals while it is not extendable (and a fortiori not weakly-inner) in some (irreducible) representation of $\mathfrak{A}$ on $\mathfrak{L}_{2}([0,1), \mu)$, where $\mu(S)$ is the number of dyadic rationals in $S$ and $[0,1)$ is partitioned by leftclosed, right-open dyadic rational subintervals. In this case, some $\iota_{\phi}(\mathfrak{U})$ is distinct from $\pi(\mathfrak{U})$. This can be used to show that $\iota_{\psi}\left(\mathfrak{H}_{0}\right)$ and $\epsilon_{\psi}\left(\mathfrak{U}_{0}\right)$ are not normal in $\alpha\left(\mathfrak{U}_{0}\right)$ for some faithful representation $\psi$ of $\mathfrak{A}_{0}(=\mathfrak{U} \oplus \mathfrak{U})$.

(b) The sum of the scalars with the compact operators gives an example of a $C^{*}$-algebra with unit for which all automorphisms are weakly-inner (in the infinite-dimensional irreducible representation) but many are not inner. In this case, $\gamma(\mathfrak{U})=\alpha(\mathfrak{U})$.

(c) With $\mathfrak{M}$ a factor of type $I_{1}$ having coupling 1 acting on separable Hilbert space and $\mathcal{C}$ the compact operators, the linear span of $\mathfrak{M}$ and $\mathfrak{C}$ is a $C^{*}$-algebra $\mathfrak{A}$. Denoting the given representation of $\mathfrak{A}$ by $\phi$, we have $\iota_{\phi}(\mathfrak{U})=\alpha(\mathfrak{A})$. Each nonscalar unitary operator $U^{\prime}$ in 
$\mathfrak{M}^{\prime}$ such that $\left\|U^{\prime}-I\right\|<1$ induces an automorphism $\alpha$ of $\mathfrak{A}$ for which $\|\alpha-\iota\|<2$; so that $\alpha$ is $\pi$-inner, yet $\alpha$ is not inner.

(d) Denoting the algebra of $n \times n$ matrices by $\mathfrak{M}_{n}$ and by $C(X)$ the algebra, under pointwise operations, of complex-valued continuous functions on the compact-Hausdorff space $X$, provides us with a class of $C^{*}$-algebras $C(X) \otimes \mathfrak{M}_{n}(=\mathfrak{U})$ for which $\gamma(\mathfrak{U}) \subseteq \iota_{0}(\mathfrak{U}) \subseteq \pi(\mathfrak{U})$ $\subseteq \alpha(\mathfrak{H})$. The group of automorphisms of $\mathfrak{U}$ which leave its center $\bar{C}(X) \otimes\{\lambda I\}$ elementwise fixed coincides with $\pi(\mathfrak{U})$. Moreover $\pi(\mathfrak{A})$ is isomorphic to the group of continuous mappings of $X$ into $\alpha\left(\mathfrak{M}_{n}\right)$ (which is $U(n) / T_{1}$, where $U(n)$ is the $n$-dimensional unitary group and $T_{1}$, its center, is the circle group). The group of mappings which "lift" from $U(n) / T_{1}$ to the bundle space $U(n)$ coincides with $\iota_{0}(\mathfrak{H})$; and $\gamma(\mathfrak{A})$ is the subgroup consisting of those mappings into $U(n) / T_{1}$ homotopic to the constant mapping (onto the unit $T_{1}$ ). Thus $\pi(\mathfrak{A}) / \gamma(\mathfrak{U})$ is the group of homotopy classes of mappings of $X$ into $U(n) / T_{1}$ and $\iota_{0}(\mathfrak{A}) / \gamma(\mathfrak{U})$ those classes which can be lifted to $U(n)$.

Since $U(n) / T_{1}$ is isomorphic to $S U(n) / \boldsymbol{Z}_{n}$, where $\boldsymbol{Z}_{n}$, the center of $S U(n)$, is the group of $n$th roots of unity, $\pi_{1}\left(U(n) / T_{1}\right) \approx Z_{n}$; and the bundle $\left\{U(n), p, U(n) / T_{1}, T_{1}, T_{1}\right\}$ ( $p$ the natural projection of $U(n)$ onto $U(n) / T_{1}$ ) does not have a cross section (since $\pi_{1}(U(n)) \approx Z$ ). Taking $X$ as $U(n) / T_{1}$, the identity mapping cannot be lifted so that $\iota_{0}(\mathfrak{U}) \neq \pi(\mathfrak{U})$. The mapping $U \boldsymbol{Z}_{n} \rightarrow U^{n} T_{1}$ of $S U(n) / \boldsymbol{Z}_{n}$ into $U(n) / T_{1}$ can be shown to be essential by using $\pi_{3}(U(n)) \approx \boldsymbol{Z}$ and the exactness of the homtopy sequence of our bundle. Thus $\gamma(\mathfrak{U}) \neq \iota_{0}(\mathfrak{H})$.

With $X$ taken as $T_{1}$, each mapping of $X$ into $U(n) / T_{1}$ can be lifted to $U(n)$ since the fibre is arcwise connected; so that $\iota_{0}(\mathfrak{U})=\pi(\mathfrak{A})$. Using a generator of $\pi_{1}\left(U(n) / T_{1}\right)$, we have an essential mapping of $X$ into $U(n) / T_{1}$, so that $\gamma(\mathfrak{U}) \neq \iota_{0}(\mathfrak{U})$.

Taking $X$ to be the 2-skeleton of a simplicial decomposition of $U(2) / T_{1}$ (homeomorphic to projective 3-space), we have $H^{1}(X, Z)$ is 0 ; so that each mapping of $X$ into $T_{1}$ is inessential (as is each mapping of $X$ into $S^{3}$ ). Thus each mapping of $X$ into $U(2)$ (homeomorphic to $\left.T_{1} \times S^{3}\right)$ is inessential, and $\iota_{0}(\mathfrak{U})=\gamma(\mathfrak{U})$. However, the identity mapping of $X$ onto itself does not lift since $\pi_{2}\left(T_{1}\right)=0$, and there would be no obstruction to extending this cross section over $X$ to one over $U(2) / T_{1}$ (while we have seen that these bundles do not have cross sections).

Denoting by $\mathfrak{A}_{m, n}$ the $C^{*}$-algebra $C\left(S^{m}\right) \otimes \mathfrak{M}_{n}$, universal bundle techniques allow us to show that $\iota_{0}\left(\mathfrak{A}_{m, n}\right)=\pi\left(\mathfrak{A}_{m, n}\right)$ for all $m, n$ $=1,2, \cdots$; and Bott's periodicity theorem tells us that $\gamma\left(\mathfrak{A}_{m, n}\right)$ $=\pi\left(\mathfrak{A}_{m, n}\right)$ for even $m<2 n$ while $\pi\left(\mathfrak{A}_{m, n}\right) / \gamma\left(\mathfrak{A}_{m, n}\right) \approx \boldsymbol{Z}$ for odd $m \neq 1$ less than $2 n$. 


\section{BIBLIOGRAPHY}

1. R. Blattner, Automorphic group representations, Pacific J. Math. 8 (1958), 665-677.

2. L. Gardner, An invariance theorem for representations of Banach algebras, Proc. Amer. Math. Soc. 16 (1965), 983-986.

3. R. Kadison, Derivations of operator algebras, Ann. of Math. 83 (1966), 280-293.

4. R. Kadison and J. Ringrose, Derivations of operator group algebras, Amer. J. Math. 88 (1966), 562-576.

5. S. Sakai, Derivations of $W^{*}$-algebras, Ann. of Math. 83 (1966), 273-279.

University of Pennsylvania and

UNIVERSITY OF NEWCASTLE, ENGLAND 